\title{
Combining High-Speed Video Imaging and Cryo-SEM to Study Droplet Impact Freezing on an Extremely Cold Surface
}

\author{
Dong Song ${ }^{1}$, Chang-Hwan Choi $^{1}$ and Tsengming Chou $^{2}$ \\ 1. Dept. of Mechanical Engineering, Stevens Institute of Technology, Hoboken, NJ. \\ 2. Laboratory of Multiscale Imaging, Stevens Institute of Technology, Hoboken, NJ.
}

Impact freezing of water droplets on cold surfaces is a universal phenomenon [1-3]. However, the mechanisms are still poorly understood, especially freezes at extremely low temperature [4]. When a drop of water impacting on extreme cold surfaces, i.e. surface temperature lower than $-80^{\circ} \mathrm{C}$, it takes more than two times longer than the one impacting at $-50^{\circ} \mathrm{C}$ surface to complete the freezing. Concurrently, evaporation and condensation accompany the freezing process and a layer of frost forms between the substrate and the frozen ice. This frozen ice also suffers a large thermal contraction and it breaks up into pieces spontaneously at the end of freezing. To study this freezing phenomenon, we use a high-speed camera (VFC-1000, FOR-A) mounted in either top- or side-view position to investigate the kinetics of cooling, and a cryo-SEM (Zeiss Auriga FIB-SEM equipped with a Leica VCT-100 transfer system) to image the de-iced surfaces at a cryogenic condition. Combing high-speed video imaging and cryo-SEM allows us to study icing mechanism at various conditions including the droplet impacting on an extremely cold surface.

Figure 1A shows the schematic of the experimental setup (top-view position of high-speed camera). Under the dried nitrogen atmosphere, a droplet of water $(39 \mu \mathrm{L})$ was made to fall on a silicon (Si) substrate that was pre-cooled to $-100^{\circ} \mathrm{C}$ using a block of aluminum (Al) as the heat sink. Figure $1 \mathrm{~B}$ and $1 \mathrm{C}$ show the captured images of top- and side-views, respectively. Especially, Figure 1C shows the delaminated and flatted regions of the frozen ice. Once the entire freezing process finished, the frozen droplet broke into several pieces and the debris fell off the Si surface, leaving visible ring marks of frost on the Si wafer (Figure 1D). Then, the whole block $(\mathrm{Si}+\mathrm{Al})$ was moved to a liquid nitrogen bath inside a glove box to further cool the sample down prior to transferring to cryo-SEM.

Figure 2A shows the SEM image taken at the delaminated region (marked in Figure 1D). It reveals a thick layer of frost. Since the experiment was performed under a dry condition, there must be a source of water in order to form this type of condensation. We observed a strong evaporation of water (plume of smoke as shown in Figure 2B) generated while the room-temperature water droplet impacted on the extreme cold surface. These vapors condensed back to form the frost on substrate. Based on the experimental observation, Figure $2 \mathrm{C}$ illustrates a mechanistic model of ice formation, suggesting weak adhesion of frozen ice to the surface due to the small contact area. Once the ice breaks up because of the thermal contraction, debris falls off spontaneously [5].

References:

[1] G. Graeber et al, "Spontaneous self-dislodging of freezing water droplets and the role of wettability," Proceedings of the National Academy of Sciences (2017).

[2] E. B. Moore, and V. Molinero, Nature 479 (2011), p. 506.

[3] J. de Ruiter, D. Soto, and K. K. Varanasi, "Self-peeling of impacting droplets," Nat Phys advance online publication (2017). 
[4] A. Bushby et al, Nature Protocols 6(6) (2011), p.845.

[5] This research project used microscopy resources within the Laboratory for Multiscale Imaging at Stevens Institute of Technology, and it was supported by the National Science Foundation (CMMI1537474).



Glove box

Figure 1. 1A shows the experimental setup. Using the video camera, we can image the drop impact freezing before it breaks up (Figure 1B). The zoom-in side-view image (Figure 1C) reveals the transition from the delaminated region to the flatted region of the frozen ice droplet. Prior to loading the sample for cryo-SEM imaging, we can identify the regions of the frost on the substrate (Figure 1D).
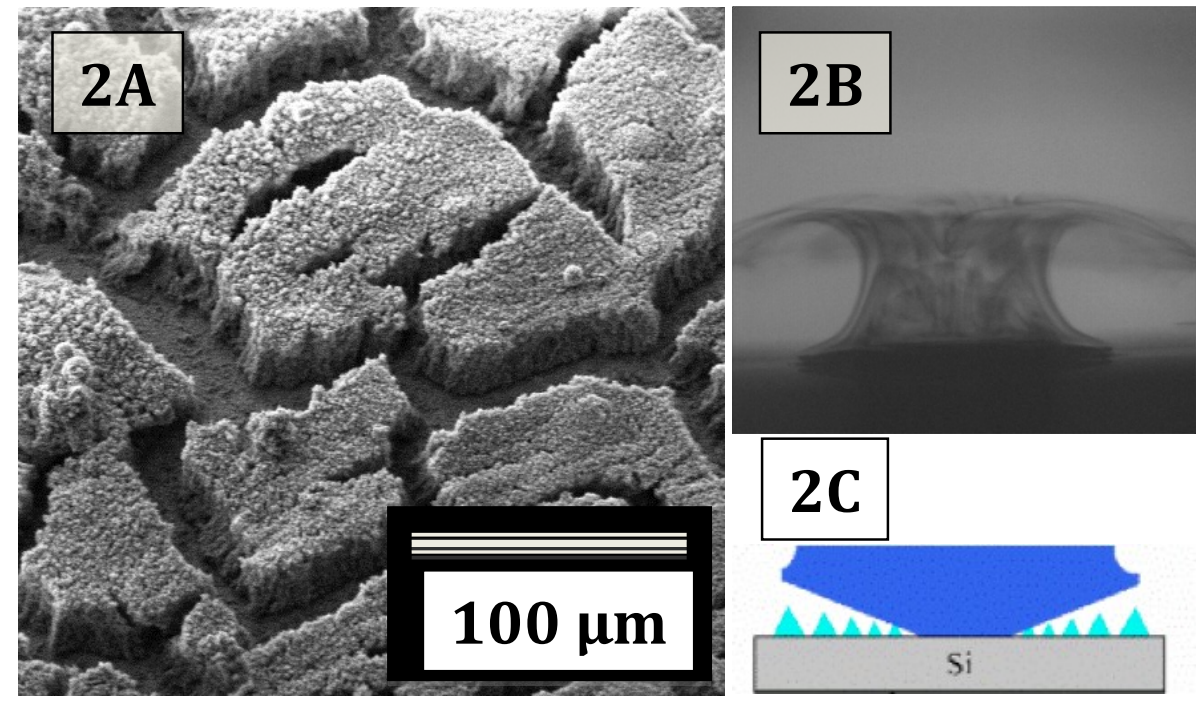

Figure 2. $2 \mathrm{~A}$ shows a thick layer of frost at the marked area of Figure 1D using cryoSEM. Figure 2B shows a plume of smoke observed during the freezing. Figure $2 \mathrm{C}$ is the proposed ice formation model for the case of droplet impact freezing on an extremely cold surface. 